\title{
Can Tsallis Distribution Fit All the Particle Spectra Produced at RHIC and LHC?
}

\author{
H. Zheng ${ }^{1}$ and Lilin $\mathrm{Zhu}^{2}$ \\ ${ }^{1}$ INFN, Laboratori Nazionali del Sud, Via Santa Sofia 62, 95123 Catania, Italy \\ ${ }^{2}$ Department of Physics, Sichuan University, Chengdu 610064, China
}

Correspondence should be addressed to H. Zheng; zheng@lns.infn.it

Received 24 April 2015; Revised 15 June 2015; Accepted 9 July 2015

Academic Editor: Xiaochun He

Copyright $\odot 2015 \mathrm{H}$. Zheng and L. Zhu. This is an open access article distributed under the Creative Commons Attribution License, which permits unrestricted use, distribution, and reproduction in any medium, provided the original work is properly cited. The publication of this article was funded by $\mathrm{SCOAP}^{3}$.

\begin{abstract}
The Tsallis distribution has been tested to fit all the particle spectra at mid-rapidity from central events produced in $\mathrm{d}+\mathrm{Au}, \mathrm{Cu}+\mathrm{Cu}$, and $\mathrm{Au}+\mathrm{Au}$ collisions at RHIC and $\mathrm{p}+\mathrm{Pb}, \mathrm{Pb}+\mathrm{Pb}$ collisions at LHC. Even though there are strong medium effects in $\mathrm{Cu}+\mathrm{Cu}$ and $\mathrm{Au}+\mathrm{Au}$ collisions, the results show that the Tsallis distribution can be used to fit most of particle spectra in the collisions studied except in $\mathrm{Au}+\mathrm{Au}$ collisions where some deviations are seen for proton and $\Lambda$ at low $p_{T}$. In addition, as the Tsallis distribution can only fit part of the particle spectra produced in $\mathrm{Pb}+\mathrm{Pb}$ collisions where $p_{T}$ is up to $20 \mathrm{GeV} / \mathrm{c}$, a new formula with one more fitting degree of freedom is proposed in order to reproduce the entire $p_{T}$ region.
\end{abstract}

\section{Introduction}

The heavy-ion collision experiments at RHIC and LHC give us the opportunity to study the phase transition from nuclear matter to quark gluon plasma (QGP), the collective motion, the nuclear medium effects, and so on. The particle spectrum is one of the basic quantities measured in experiments to address the questions raised in such studies. Recently, the Tsallis distribution has attracted the attention of many theorists and experimentalists in high energy heavy-ion collisions [1-25]. It has been applied to particle spectra produced in different reaction systems, from $\mathrm{pp}, \mathrm{pA}$ to $\mathrm{AA}$, to understand the particle production mechanism and extract physical quantities, for example, temperature [2-4, 7, 12-14, 17-21, 24] and chemical potential [26]. In pp collisions, the excellent ability to fit the spectra of identified hadrons and charged particles in a large range of $p_{T}$ up to $200 \mathrm{GeV} / \mathrm{c}$ is quite impressive [2, 23-25]. A systematic investigation of particle spectra in $\mathrm{p}+\mathrm{p}$ collisions at RHIC and LHC has been conducted in [2]. The results show that the Tsallis distribution can fit all the particle spectra at different energies in $p+p$ collisions. A possible cascade particle production mechanism is proposed. Recently, a Tsallis distribution scaling function was found for charged hadron spectra in $p+p$ and $p+\bar{p}$ collisions [1]. Comparing to nucleus-nucleus collisions, the pp collision is very simple. It has been used as a baseline for nucleus-nucleus collisions. A nuclear modification factor $R_{\mathrm{pA}}$ or $R_{\mathrm{AA}}$ was proposed to show the nuclear medium effects in pA or AA collisions referring to pp collisions $[5,10-12,27-$ 34]. The nuclear modification factor different from unity is a manifestation of medium effects. Many authors have successfully applied Tsallis distribution to fit particle spectra in $\mathrm{pA}$ and $\mathrm{AA}$ collisions even though the spectra were affected by nuclear medium modification $[3-8,10,11,18,21]$. We also notice that many works only show the small $p_{T}$ part of the particle spectra, while the exponential distribution also can fit the low $p_{T}$ region $[11,35]$. It should cover all $p_{T}$ regions of particle spectra, available in experiment, in order to show the advantage and/or the fitting power of the Tsallis distribution. In recent years, the experimental groups at RHIC and LHC have published the wide $p_{T}$ range of particle spectra for different particles in different reaction systems. Such data allow us to conduct the systematic study of particle spectra in 
heavy-ion collisions at RHIC and LHC using the Tsallis distribution, as we have done for $\mathrm{p}+\mathrm{p}$ collisions [2].

In this work, we would like to test whether the Tsallis distribution can fit all the particle spectra produced at RHIC and LHC, which can help us to understand the particle production mechanism. Before we start to conduct our investigation, we can get some clues to estimate whether it can fit the particle spectrum or not from the nuclear modification factor. If $R_{\mathrm{pA}}$ or $R_{\mathrm{AA}}$ is flat for the whole $p_{T}$ region, according to its definition, this means that the particle spectrum is similar in shape and only differs in magnitude to the one in $p+p$ collisions. Based on the previous studies $[1,2]$, we are sure that the Tsallis distribution can fit the particle spectrum since it can fit all the particle spectra produced in $\mathrm{p}+\mathrm{p}$ collisions, especially up to extremely high $p_{T}[2,23-25]$. In the $\mathrm{pA}$ reactions, $R_{\mathrm{pA}}$ are flat and very close to 1 for most of the produced particles at different centralities $[5,10,11,27,29$, 34], while in the AA collisions the nuclear medium effects play an important role. $R_{\mathrm{AA}}$ increases from the most central collisions to peripheral collisions [11, 12, 28, 30-33]. Since the nuclear modification factors of different particles are almost 1 in peripheral heavy-ion collisions [5, 10-12, 27-34], as we discussed, Tsallis distribution should be able to fit the particle spectra. Therefore we will only focus on the particle spectra at the most central collisions where the Tsallis distribution may not fit all of them. We have collected data of particle spectra from $\mathrm{d}+\mathrm{Au}, \mathrm{Cu}+\mathrm{Cu}$, and $\mathrm{Au}+\mathrm{Au}$ collisions at RHIC and $\mathrm{p}$ $+\mathrm{Pb}, \mathrm{Pb}+\mathrm{Pb}$ collisions at $\mathrm{LHC}$ and select the data for most of the particles with the highest $p_{T}>5 \mathrm{GeV}$ to conduct this study.

The paper is organized as follows. In Section 2, we show different versions of the Tsallis distribution used in the literature. More details can be found in [2]. We also give the form of Tsallis distribution used in our analysis. In Section 3, we show our results of particle spectra from $\mathrm{d}+\mathrm{Au}, \mathrm{p}+\mathrm{Pb}, \mathrm{Cu}$ $+\mathrm{Cu}, \mathrm{Au}+\mathrm{Au}$, and $\mathrm{Pb}+\mathrm{Pb}$. Another distribution is proposed to fit the particle spectra in $\mathrm{Pb}+\mathrm{Pb}$ collisions since Tsallis distribution can only fit part of the particle spectra in the case. A brief conclusion is given in Section 4 .

\section{Tsallis Distributions}

In the literature, several versions of Tsallis distribution with different arguments can be found [2-25]. The asymptotic behaviors of these distributions at low and high $p_{T}$ limits can be found in [2]. We only briefly show them here.

The STAR [9] and PHENIX [5, 12] Collaborations at RHIC along with ALICE $[13,14]$ and CMS [15] Collaborations at LHC adopted this form of Tsallis distribution:

$$
\begin{aligned}
& E \frac{d^{3} N}{d p^{3}}=\frac{1}{2 \pi p_{T}} \frac{d^{2} N}{d y d p_{T}} \\
& \quad=\frac{d N}{d y} \frac{(n-1)(n-2)}{2 \pi n C[n C+m(n-2)]}\left(1+\frac{m_{T}-m}{n C}\right)^{-n},
\end{aligned}
$$

where $m_{T}=\sqrt{p_{T}^{2}+m^{2}}$ is the transverse mass and $m$ is the mass of the particle. $d N / d y, n$, and $C$ are fitting parameters.

In $[8,19-22,36]$, the following Tsallis form is used:

$$
\begin{aligned}
& E \frac{d^{3} N}{d p^{3}} \\
& \quad=g V \frac{m_{T} \cosh y}{(2 \pi)^{3}}\left[1+(q-1) \frac{m_{T} \cosh y-\mu}{T}\right]^{q /(1-q)},
\end{aligned}
$$

based on thermodynamic consistency arguments. Where $g$ is the degeneracy of the particle, $V$ is the volume, $y$ is the rapidity, $\mu$ is the chemical potential, $T$ is the temperature, and $q$ is a parameter. In (2), there are four parameters $V$, $\mu, T$, and $q . \mu$ was assumed to be 0 in $[19-21,36]$ which is a reasonable assumption because the energy is high enough and the chemical potential is small compared to temperature. In the mid-rapidity $y=0$ region, (2) is reduced to

$$
E \frac{d^{3} N}{d p^{3}}=g V \frac{m_{T}}{(2 \pi)^{3}}\left[1+(q-1) \frac{m_{T}}{T}\right]^{q /(1-q)} .
$$

In $[4,21],(2)$ has been rewritten as

$$
\begin{aligned}
& \frac{d N}{m_{T} d m_{T}} \\
& =C \int_{-Y}^{Y} \cosh y d y m_{T}\left[1+(q-1) \frac{m_{T} \cosh y}{T}\right]^{q /(1-q)},
\end{aligned}
$$

to take into account the width of the corresponding rapidity distribution of the particles.

In [17], Sena and Deppman applied the nonextensive formalism to obtain the probability of particle with momentum $p_{T}$ as

$$
\begin{aligned}
& \frac{1}{\sigma} \frac{d \sigma}{d p_{T}} \\
& =c p_{T} \int_{0}^{\infty} d p_{L}\left[1+(q-1) \beta \sqrt{p_{L}^{2}+p_{T}^{2}+m^{2}}\right]^{-q /(q-1)},
\end{aligned}
$$

where $c$ is the normalization constant, $q$ is a parameter, $\beta=$ $1 / T$, and $m$ is the mass of particle. With the approximation $p_{T}$ very large compared to $p_{L}$ and $m$ [37], (5) can be rewritten as

$$
\begin{gathered}
\frac{1}{\sigma} \frac{d \sigma}{d p_{T}}=c[2(q-1)]^{-1 / 2} B\left(\frac{1}{2}, \frac{q}{q-1}-\frac{1}{2}\right) \\
\cdot u^{3 / 2}[1+(q-1) u]^{-q /(q-1)+1 / 2},
\end{gathered}
$$

where $u=p_{T} / T$ and $B(x, y)$ is the beta-function.

In [24], Wong and Wilk proposed a new form of the Tsallis distribution function to take into account the rapidity cut:

$$
\left(E \frac{d^{3} N}{d p^{3}}\right)_{|\eta|<a}=\int_{-a}^{a} d \eta \frac{d y}{d \eta}\left(\frac{d^{3} N}{d p^{3}}\right),
$$


where

$$
\frac{d y}{d \eta}\left(\eta, p_{T}\right)=\sqrt{1-\frac{m^{2}}{m_{T}^{2} \cosh ^{2} y}}
$$

with

$$
\begin{aligned}
y & =\frac{1}{2} \ln \left[\frac{\sqrt{p_{T}^{2} \cosh ^{2} \eta+m^{2}}+p_{T} \sinh \eta}{\sqrt{p_{T}^{2} \cosh ^{2} \eta+m^{2}}-p_{T} \sinh \eta}\right], \\
\frac{d^{3} N}{d p^{3}} & =C \frac{d N}{d y}\left(1+\frac{E_{T}}{n T}\right)^{-n}, \quad E_{T}=m_{T}-m,
\end{aligned}
$$

where $C(d N / d y)$ is assumed to be a constant.

In [2], we have obtained

$$
\left(E \frac{d^{3} N}{d p^{3}}\right)_{|\eta|<a}=A\left(1+\frac{E_{T}}{n T}\right)^{-n}
$$

where $A, n$, and $T$ are the fitting parameters. This is equivalent to (1) but in a simpler form. We adopt (10) to do the analysis here. We notice that (10) has been used by the CMS Collaboration $[16,38]$ and by Wong et al. in their recent paper [25]. The STAR Collaboration has also applied a formula which is very close to (10) [29].

\section{Results}

We have selected the data of particle spectra from the most central collisions with the highest $p_{T}>5 \mathrm{GeV}$ for most of the particles in $\mathrm{d}+\mathrm{Au}, \mathrm{p}+\mathrm{Pb}, \mathrm{Cu}+\mathrm{Cu}, \mathrm{Au}+\mathrm{Au}$, and $\mathrm{Pb}+\mathrm{Pb}$ at RHIC and LHC. We fit the center values of the experimental points. The fit metric used is defined by

$$
M^{2}=\sum_{i}\left[1-\frac{y_{i}(\text { fit })}{y_{i}(\text { data })}\right]^{2} .
$$

As we discussed before, the Tsallis distribution should be able to fit the particle spectra from $\mathrm{d}+\mathrm{Au}$ and $\mathrm{p}+\mathrm{Pb}$. One good example has been shown in $[4,5]$ for $K_{S}^{0}$ and $K^{0 *}$ in d + $\mathrm{Au}$ at $\sqrt{s_{N N}}=200 \mathrm{GeV}$ where their spectra can be obtained by multiplying the particle spectra in $\mathrm{p}+\mathrm{p}$ collisions with $N_{\text {coll }}$. In Figures 1 and 2, our results for $\mathrm{d}+\mathrm{Au}$ at $\sqrt{s_{N N}}=$ $200 \mathrm{GeV}$ and $\mathrm{p}+\mathrm{Pb}$ at $\sqrt{s_{N N}}=5.02 \mathrm{TeV}$ using (10) have been shown. In order to see the agreement between the data and the Tsallis distribution in linear scale, a ratio data/fit is defined. As shown in Figures 1 and 2, the fits for all particles are good. For the left collision systems, we also do the same comparisons. We would like to emphasize that $p_{T}$ of charged particle spectrum is up to $45 \mathrm{GeV} / \mathrm{c}$.

Now let us turn to the AA collisions. First we use Tsallis distribution Equation (10) to fit the particle spectra in $\mathrm{Au}+$ Au collisions at $\sqrt{s_{N N}}=62.4 \mathrm{GeV}$ in Figure 3. All the particle spectra are well fitted except the proton spectrum at $p_{T}<$ $1 \mathrm{GeV} / \mathrm{c}$. This makes a little difference of AA collisions from $\mathrm{p}+\mathrm{p}$ collisions. We want to check whether this deviation will

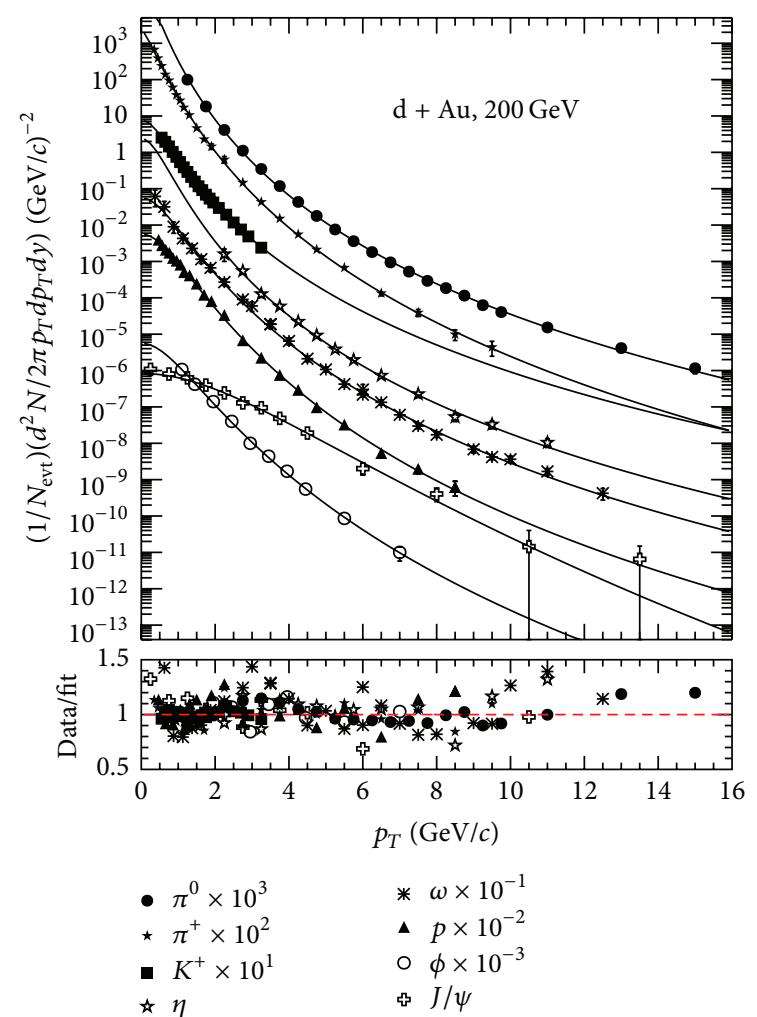

Figure 1: The data are from $[10,11,27,39-42]$ for $\mathrm{d}+\mathrm{Au}$ at $\sqrt{s_{N N}}=200 \mathrm{GeV}$. The curves are the analytical results with Tsallis distribution Equation (10). The corresponding fitting parameters and $\chi^{2} /$ ndf are given in Table 1 . For a better visualization both the data and the analytical curves have been scaled by a constant as indicated. The ratios of data/fit are shown at the bottom.

become larger at higher colliding energy in AA collisions. We considered the particle spectra from $\mathrm{Cu}+\mathrm{Cu}$ collisions at $\sqrt{s_{N N}}=200 \mathrm{GeV}$. The results are shown in Figure 4. The fitting with (10) for different particle spectra is very well. But we do not know whether there is deviation or not for proton at low $p_{T}$ since the data for $p_{T}<3 \mathrm{GeV} / \mathrm{c}$ are not available. Fortunately, the data for different particle spectra at low $p_{T}$ in $\mathrm{Au}+\mathrm{Au}$ collisions at $\sqrt{s_{N N}}=200 \mathrm{GeV}$ are given. In Figure 5, one can see the deviations of particle spectra of proton and $\Lambda$ at low $p_{T}$ from the Tsallis distribution Equation (10). While a deviation is observed for proton at $p_{T}<2 \mathrm{GeV} / \mathrm{c}$ which becomes a little larger than the one in $\mathrm{Au}+\mathrm{Au}$ at $\sqrt{s_{N N}}=62.4 \mathrm{GeV}$, all other particle spectra are well fitted. This makes us curious to fit the particle spectra in $\mathrm{Pb}+\mathrm{Pb}$ collisions at $\sqrt{s_{N N}}=2.76 \mathrm{TeV}$. With the successful running at LHC, the identified hadron particle spectra data in $\mathrm{Pb}+\mathrm{Pb}$ collisions at $\sqrt{s_{N N}}=2.76 \mathrm{TeV}$ are available up to $20 \mathrm{GeV} / \mathrm{c}$. The data satisfy two criteria. One is that there are strong nuclear medium effects in $\mathrm{Pb}+\mathrm{Pb}$ collisions which can be seen from $R_{\mathrm{PbPb}}$ and the other is that the transverse momenta of the particles reach high values. This gives us an opportunity to test the fitting power of the Tsallis distribution. When we use Tsallis distribution Equation (10) to fit pion spectrum, we 
TABLE 1: The fitting parameters and the corresponding $\chi^{2} /$ ndf for various particles in different collision systems with Tsallis distribution Equation (10).

\begin{tabular}{|c|c|c|c|c|c|c|}
\hline System & Particle & Centrality & $A$ & $T(\mathrm{GeV})$ & $n$ & $\chi^{2} / \mathrm{ndf}$ \\
\hline \multirow{9}{*}{$\begin{array}{l}\mathrm{d}+\mathrm{Au} \\
\sqrt{s_{N N}}=200 \mathrm{GeV}\end{array}$} & $\gamma$ & Minimum bias & 1274.39 & 0.108 & 7.48 & $30.66 / 21$ \\
\hline & $\pi^{0}$ & $0-20 \%$ & 54.08 & 0.130 & 9.70 & $15.46 / 18$ \\
\hline & $\pi^{+}$ & $0-20 \%$ & 21.60 & 0.173 & 11.56 & $10.74 / 21$ \\
\hline & $K^{+}$ & $0-20 \%$ & 0.776 & 0.214 & 8.89 & $1.54 / 18$ \\
\hline & $\eta$ & $0-20 \%$ & 2.403 & 0.163 & 9.57 & $6.75 / 10$ \\
\hline & $\omega$ & $0-20 \%$ & 0.565 & 0.224 & 10.53 & $18.08 / 24$ \\
\hline & $p$ & $0-20 \%$ & 0.568 & 0.221 & 11.97 & $11.60 / 20$ \\
\hline & $\phi$ & $0-20 \%$ & 0.0563 & 0.270 & 13.63 & $3.57 / 7$ \\
\hline & $J / \psi$ & $0-20 \%$ & $8.30 E-7$ & 0.582 & 26.91 & $15.67 / 10$ \\
\hline \multirow{4}{*}{$\begin{array}{l}\mathrm{p}+\mathrm{Pb} \\
\sqrt{s_{N N}}=5.02 \mathrm{TeV}\end{array}$} & Charged & Minimum bias & 33.32 & 0.182 & 7.00 & $501.03 / 57$ \\
\hline & $K_{s}^{0}$ & $0-5 \%$ & 1.537 & 0.302 & 9.16 & $15.12 / 31$ \\
\hline & $p$ & $0-5 \%$ & 0.536 & 0.449 & 22.49 & $6.53 / 36$ \\
\hline & $\Lambda$ & $0-5 \%$ & 0.323 & 0.469 & 19.47 & $21.13 / 17$ \\
\hline \multirow{6}{*}{$\begin{array}{l}\mathrm{Au}+\mathrm{Au} \\
\sqrt{s_{N N}}=62.4 \mathrm{GeV}\end{array}$} & $\pi^{+}$ & $0-10 \%$ & 551.14 & 0.171 & 16.80 & $20.80 / 20$ \\
\hline & $K_{s}^{0}$ & $0-5 \%$ & 20.03 & 0.264 & 40.88 & $12.95 / 12$ \\
\hline & $p$ & $0-10 \%$ & 21.37 & 0.226 & 22.64 & $305.47 / 16$ \\
\hline & $\Lambda$ & $0-5 \%$ & 6.83 & 0.295 & 11039.30 & $7.26 / 9$ \\
\hline & $\Xi$ & $0-5 \%$ & 0.352 & 0.315 & 7811.73 & $6.94 / 8$ \\
\hline & $\Omega$ & $0-20 \%$ & $4.35 E-2$ & 0.308 & 3064.05 & $2.57 / 2$ \\
\hline \multirow{9}{*}{$\begin{array}{l}\mathrm{Cu}+\mathrm{Cu} \\
\sqrt{s_{N N}}=200 \mathrm{GeV}\end{array}$} & $\pi^{0}$ & $0-10 \%$ & 304.12 & 0.128 & 9.42 & $38.83 / 21$ \\
\hline & $\pi^{+}$ & $0-10 \%$ & 1016.57 & 0.111 & 9.45 & $7.16 / 8$ \\
\hline & $K_{s}^{0}$ & $0-10 \%$ & 15.89 & 0.198 & 12.19 & $51.37 / 19$ \\
\hline & $\omega$ & $0-20 \%$ & 51.79 & 0.139 & 9.48 & $1.21 / 4$ \\
\hline & $p$ & $0-10 \%$ & 242.07 & 0.107 & 9.90 & $0.12 / 5$ \\
\hline & $\phi$ & $0-10 \%$ & 1.09 & 0.226 & 12.08 & $7.02 / 8$ \\
\hline & $\Lambda$ & $0-10 \%$ & 2.04 & 0.297 & 35.35 & $121.47 / 16$ \\
\hline & $\Xi$ & $0-10 \%$ & 0.199 & 0.326 & 42.47 & $6.25 / 7$ \\
\hline & $J / \Psi$ & $0-20 \%$ & $7.98 E-6$ & 0.399 & 8.16 & $3.60 / 7$ \\
\hline \multirow{10}{*}{$\begin{array}{l}\mathrm{Au}+\mathrm{Au} \\
\sqrt{s_{N N}}=200 \mathrm{GeV}\end{array}$} & $\gamma$ low $p_{T}$ & $0-20 \%$ & 109.59 & 0.184 & 19.24 & $4.10 / 8$ \\
\hline & $\gamma$ high $p_{T}$ & $0-5 \%$ & 4.64 & 0.187 & 7.85 & $15.44 / 14$ \\
\hline & $\pi^{0}$ & $0-10 \%$ & 18.68 & 0.191 & 9.06 & $12.36 / 12$ \\
\hline & $\pi^{+}$ & $0-10 \%$ & 1165.01 & 0.138 & 10.50 & $251.34 / 28$ \\
\hline & $K_{s}^{0}$ & $0-5 \%$ & 49.56 & 0.213 & 13.99 & $153.73 / 18$ \\
\hline & $\eta$ & $0-20 \%$ & 4978.81 & 0.066 & 8.16 & $4.03 / 7$ \\
\hline & $p$ & $0-12 \%$ & 312.81 & 0.118 & 9.50 & $54866.3 / 26$ \\
\hline & $\phi$ & $0-10 \%$ & 3.44 & 0.236 & 13.70 & $7.85 / 7$ \\
\hline & $\Lambda$ & $0-5 \%$ & 31.74 & 0.215 & 18.91 & $2739.02 / 18$ \\
\hline & $J / \Psi$ & $0-20 \%$ & $3.576 E-4$ & 0.221 & 7.88 & $0.83 / 3$ \\
\hline
\end{tabular}




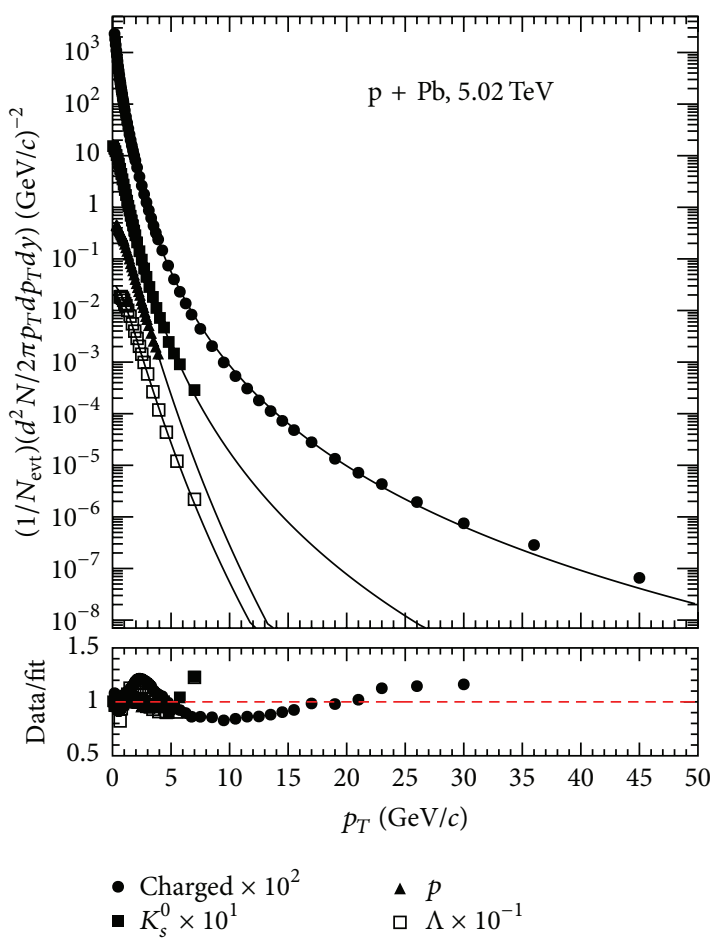

Figure 2: The data are from $[34,43]$ for $\mathrm{p}+\mathrm{Pb}$ at $\sqrt{s_{N N}}=5.02 \mathrm{TeV}$. The curves are the analytical results with Tsallis distribution Equation (10). The corresponding fitting parameters and $\chi^{2} / \mathrm{ndf}$ are given in Table 1. For a better visualization both the data and the analytical curves have been scaled by a constant as indicated. The ratios of data/fit are shown at the bottom.

find that (10) can only fit part of it. If we choose to fit the low $p_{T}$ region, (10) can fit the particle spectrum up to $10 \mathrm{GeV} / \mathrm{c}$, as shown in Figure 6 with red dashed line. The blue dotteddashed line shows the fit for high $p_{T}$ region which starts from $4 \mathrm{GeV} / \mathrm{c}$.

The exponential form equation was used to fit the particle spectra at RHIC when only the low $p_{T}$ data are available [11, 35]. With the upgrade of detectors, we have a better ability to measure the particle spectra. When the intermediate $p_{T}$ data are available, the Tsallis distribution is used to understand the particle spectra and extract physical information. The twoBoltzmann distribution was also used in [7]. But in both cases the number of free fitting parameters increases from 2 to 3. One fitting degree of freedom is increased in this transition. In [6], a double Tsallis formula was proposed to fit particle spectra obtained from central events in $\mathrm{Pb}+\mathrm{Pb}$ collisions. In this case, three fitting degrees of freedom are increased. Here we will follow the same logic of the transition from exponential distribution to Tsallis distribution to propose a new form equation to fit the particle spectra in $\mathrm{Pb}+\mathrm{Pb}$ collisions at $\sqrt{s_{N N}}=2.76 \mathrm{TeV}$ by only increasing one fitting degree of freedom. We increase the number of free fitting parameters from 3 to 4 and the proposed formula is

$$
\left(E \frac{d^{3} N}{d p^{3}}\right)_{|\eta|<a}=A \frac{e^{-(b / T) \arctan \left(E_{T} / b\right)}}{\left[1+\left(E_{T} / b\right)^{4}\right]^{c}} .
$$

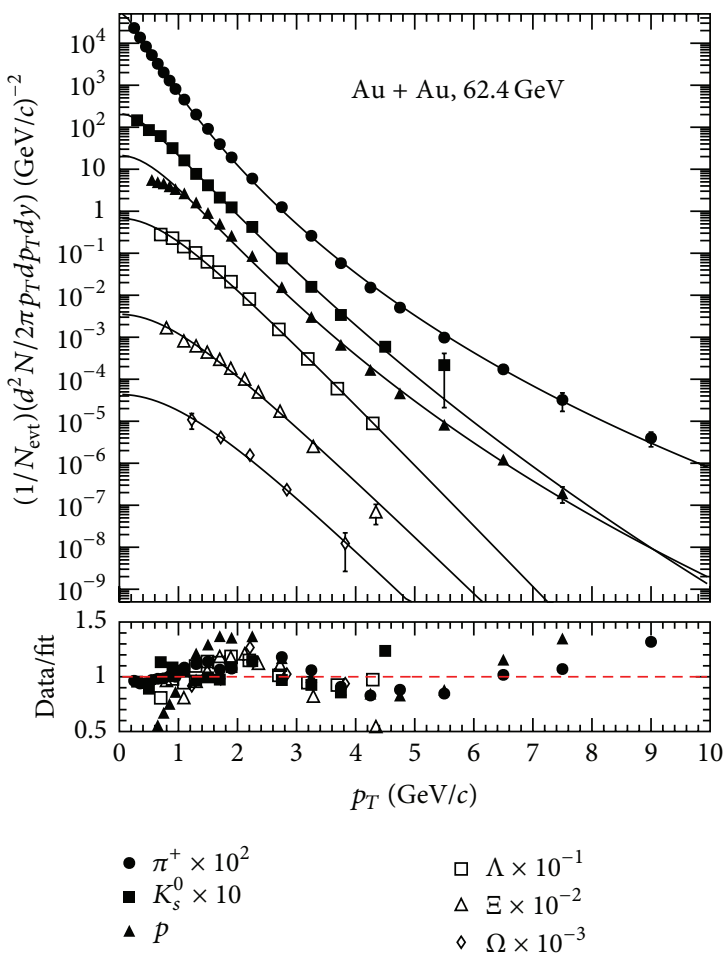

FIgURE 3: The data are from $[30,44]$ for $\mathrm{Au}+\mathrm{Au}$ at $\sqrt{s_{N N}}=$ $62.4 \mathrm{GeV}$. The curves are the analytical results with Tsallis distribution Equation (10). The corresponding fitting parameters and $\chi^{2} / \mathrm{ndf}$ are given in Table 1 . For a better visualization both the data and the analytical curves have been scaled by a constant as indicated. The ratios of data/fit are shown at the bottom (Color online).

There are four parameters $A, b, T$, and $c$. We are inspired by the solution of Fokker-Planck equation [56]. We change the power from 2 in [56] to 4 in (12) in order to fit well all the particle spectra with one equation. Figure 6 shows that the fits with (12) are excellent. We would like to mention that when $E_{T} / b \ll 1$, (12) becomes

$$
\left(E \frac{d^{3} N}{d p^{3}}\right)_{|\eta|<a} \propto e^{-E_{T} / T}
$$

and when $E_{T} / b \gg 1$,

$$
\left(E \frac{d^{3} N}{d p^{3}}\right)_{|\eta|<a} \propto p_{T}^{-4 c}
$$

Equation (12) has the same asymptotic behaviors as (10).

\section{Conclusions}

In this paper, we have tested the fitting ability of Tsallis function by fitting different particle spectra produced at the most central collisions in $\mathrm{d}+\mathrm{Au}, \mathrm{p}+\mathrm{Pb}, \mathrm{Cu}+\mathrm{Cu}, \mathrm{Au}+$ $\mathrm{Au}$, and $\mathrm{Pb}+\mathrm{Pb}$ at RHIC and LHC. The Tsallis distribution is able to fit all the particle spectra in $d+A u$ and $p+$ $\mathrm{Pb}$ collisions where the medium effects are very weak. This information can be obtained by the nuclear modification 
TABLE 2: The fitting parameters and the corresponding $\chi^{2} /$ ndf for different particles in $\mathrm{Pb}+\mathrm{Pb}$ at $\sqrt{\boldsymbol{s}_{N N}}=2.76 \mathrm{TeV}$ with (12).

\begin{tabular}{lccccccc}
\hline System & Particle & Centrality & $A$ & $T(\mathrm{GeV})$ & $b$ & $c$ & $\chi^{2} / \mathrm{ndf}$ \\
\hline & Charged $\pi$ & $0-5 \%$ & 2049.91 & 0.252 & 2.195 & 0.886 & $174.54 / 59$ \\
& Charged $K$ & $0-5 \%$ & 112.68 & 0.346 & 1.776 & 1.150 & $35.37 / 54$ \\
$\mathrm{~Pb}+\mathrm{Pb}$ & Charged $p$ & $0-5 \%$ & 10.55 & 0.710 & 1.845 & 1.605 & $42.29 / 45$ \\
$\sqrt{s_{N N}}=2.76 \mathrm{TeV}$ & $\phi$ & $0-5 \%$ & 2.11 & 0.749 & 1.281 & 1.080 & $1.84 / 4$ \\
& $\Lambda$ & $0-5 \%$ & 3.525 & 0.761 & 1.907 & 1.679 & $23.15 / 27$ \\
& $\Xi$ & $0-10 \%$ & 0.376 & 0.774 & 2.003 & 1.665 & $32.95 / 23$ \\
& $\Omega$ & $0-10 \%$ & 0.0615 & 0.658 & 2.098 & 1.324 & $2.41 / 9$ \\
\hline
\end{tabular}

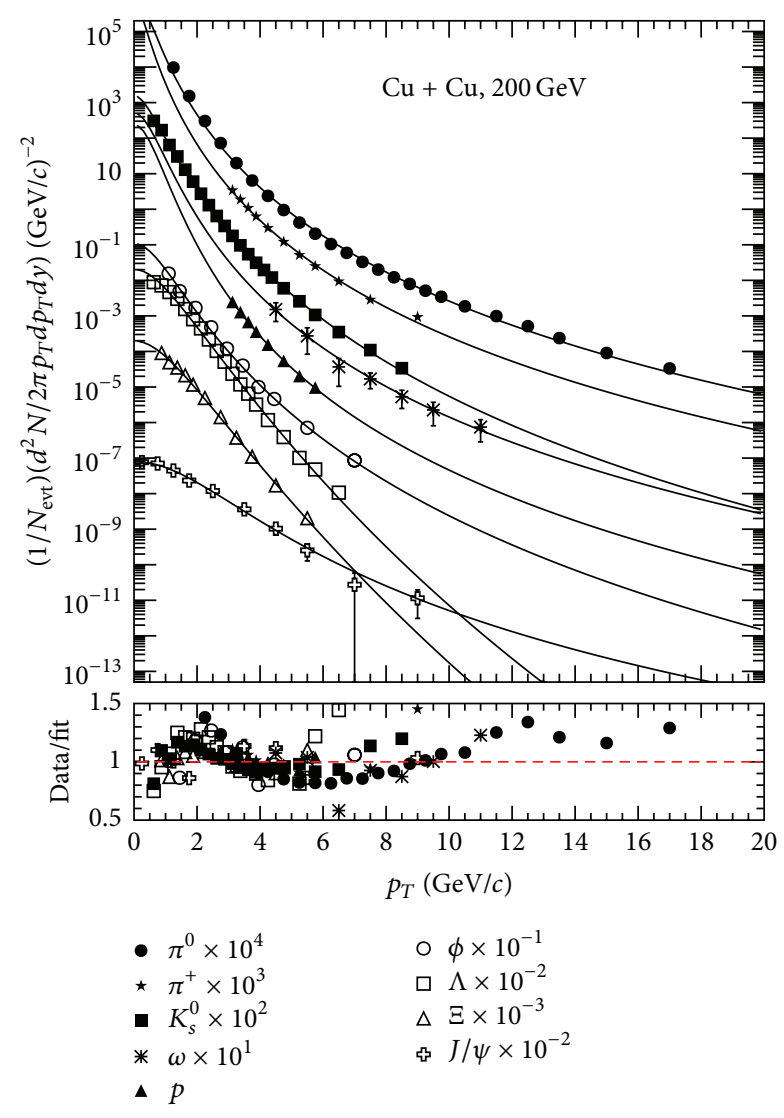

Figure 4: The data are from $[11,31,40,45-47]$ for $\mathrm{Cu}+\mathrm{Cu}$ at $\sqrt{s_{N N}}=200 \mathrm{GeV}$. The curves are the analytical results with Tsallis distribution Equation (10). The corresponding fitting parameters and $\chi^{2} / \mathrm{ndf}$ are given in Table 1 . For a better visualization both the data and the analytical curves have been scaled by a constant as indicated. The ratios of data/fit are shown at the bottom.

factor. In the AA collisions, the Tsallis distribution can fit all the particle spectra very well at RHIC energies except the little deviation observed for proton and $\Lambda$ at low $p_{T}$. However the Tsallis distribution can only fit part of the particle spectra in $\mathrm{Pb}+\mathrm{Pb}$ at $\sqrt{s_{N N}}=2.76 \mathrm{TeV}$, either in the low or in the high $p_{T}$ region. We have proposed a new formula in order to fit all the particle spectra in $\mathrm{Pb}+\mathrm{Pb}$ by increasing one fitting degree of freedom from Tsallis distribution. This follows

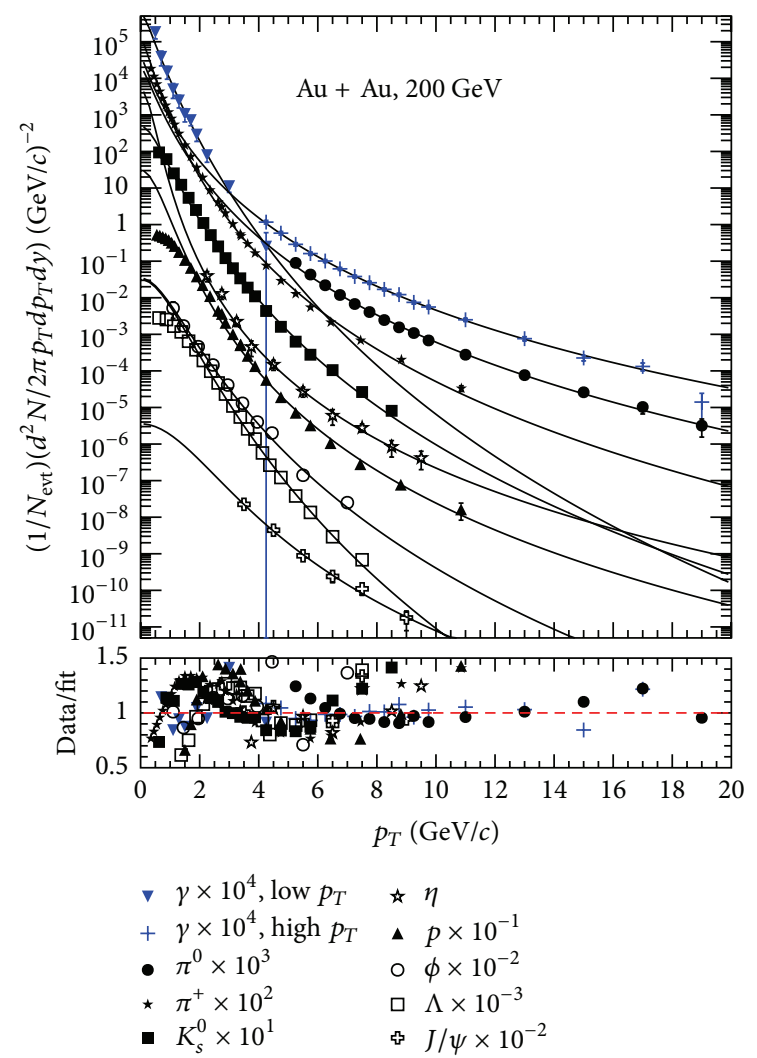

Figure 5: The data are from $[11,32,46,48-52]$ for $\mathrm{Au}+\mathrm{Au}$ at $\sqrt{s_{N N}}=200 \mathrm{GeV}$. The curves are the analytical results with Tsallis distribution Equation (10). The corresponding fitting parameters and $\chi^{2} / \mathrm{ndf}$ are given in Table 1 . For a better visualization both the data and the analytical curves have been scaled by a constant as indicated. The ratios of data/fit are shown at the bottom.

the same idea of the transition from the exponential distribution to Tsallis distribution when intermediate $p_{T}$ data are available in experiments.

According to the results in this paper and [2], we conclude that we can do the systematic analysis of particle spectra with Tsallis distribution in $\mathrm{p}+\mathrm{p}, \mathrm{pA}$ at RHIC and LHC. In the AA collisions at $p_{T}<10 \mathrm{GeV} / \mathrm{c}$, we can do the same analysis as in $\mathrm{p}+\mathrm{p}$ and $\mathrm{pA}$ at RHIC and LHC. But when we consider $\mathrm{Pb}+$ $\mathrm{Pb}$ collisions, the Tsallis distribution fails. 


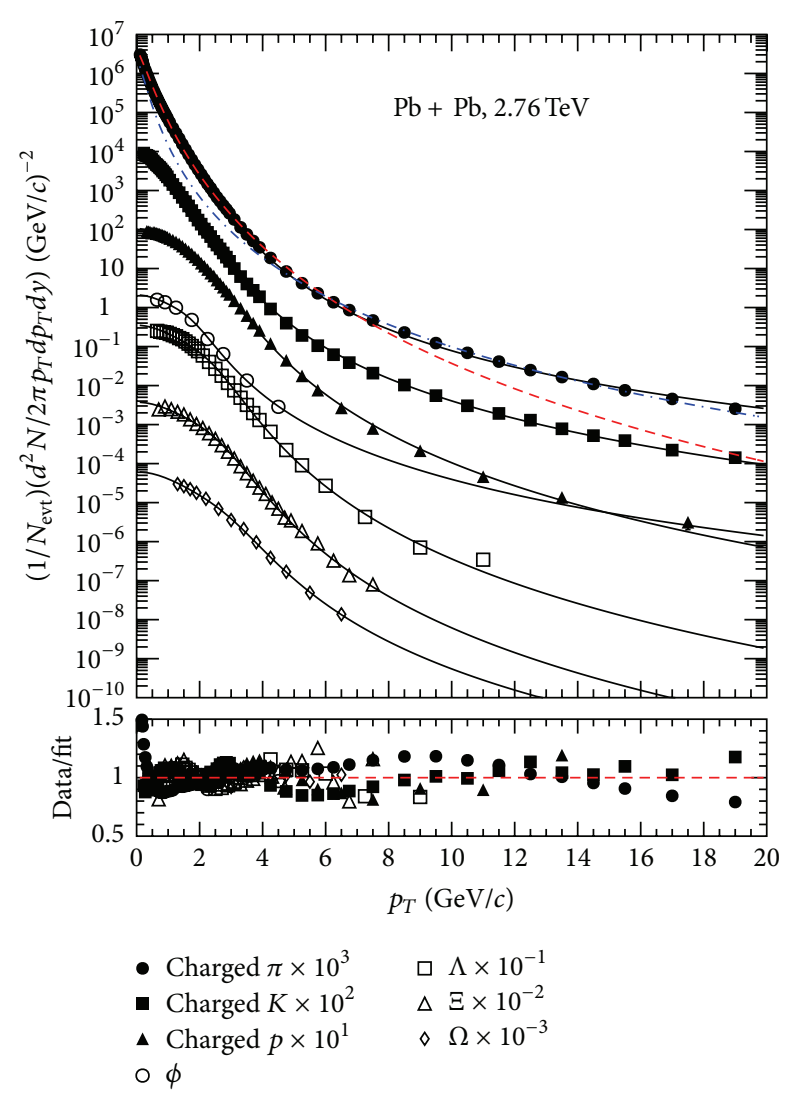

FIgURE 6: The data are from $[33,53-55]$ for $\mathrm{Pb}+\mathrm{Pb}$ at $\sqrt{\bar{s}_{N N}}=$ $2.76 \mathrm{TeV}$. The red dashed line is the results fitting the low $p_{T}$ region and the blue dotted-dashed line is the results fitting the high $p_{T}$ region using (10). The solid curves are the analytical results with (12). The corresponding fitting parameters and $\chi^{2} / \mathrm{ndf}$ are given in Table 2 . For a better visualization both the data and the analytical curves have been scaled by a constant as indicated. The ratios of data/fit are shown at the bottom.

\section{Conflict of Interests}

The authors declare that there is no conflict of interests regarding the publication of this paper.

\section{Acknowledgments}

The authors thank Dr. J. Mabiala for reading carefully their paper. This work was supported, in part, by the NSFC of China under Grant no. 11205106.

\section{References}

[1] W. C. Zhang and C. B. Yang, "Scaling behaviour of charged hadron $p_{T}$ distributions in pp and $p \bar{p}$ collisions," Journal of Physics G: Nuclear and Particle Physics, vol. 41, no. 10, Article ID 105006, 9 pages, 2014.

[2] H. Zheng, L. Zhu, and A. Bonasera, "Systematic analysis of hadron spectra in $p+p$ collisions using Tsallis distribution," http://arxiv.org/abs/1506.03156.

[3] P. K. Khandai, P. Sett, P. Shukla, and V. Singh, "System size dependence of hadron $p_{T}$ spectra in $p+p$ and $\mathrm{Au}+\mathrm{Au}$ collisions at $\sqrt{s_{N N}}=200 \mathrm{GeV}$," Journal of Physics G: Nuclear and Particle Physics, vol. 41, no. 2, Article ID 025105, 2014.

[4] B.-C. Li, G.-X. Zhang, and Y.-Y. Guo, "Transverse momentum spectra of $K_{S}^{0}$ and $K^{* 0}$ at midrapidity in $d+\mathrm{Au}, \mathrm{Cu}+\mathrm{Cu}$, and $p+p$ collisions at $\sqrt{s_{N N}}=200 \mathrm{GeV}$,' Advances in High Energy Physics, vol. 2015, Article ID 684950, 8 pages, 2015.

[5] A. Adare, S. Afanasiev, C. Aidala et al., "Measurement of $K_{s}^{0}$ and $K^{* 0}$ in $p+p, d+\mathrm{Au}$ and $\mathrm{Cu}+\mathrm{Cu}$ collisions at $\sqrt{s_{N N}}=200 \mathrm{GeV}$," Physical Review C, vol. 90, no. 5, Article ID 054905, 18 pages, 2014.

[6] M. Rybczyn’ski, G. Wilk, and Z. Włodarczyk, "System size dependence of the log-periodic oscillations of transverse momentum spectra," EPJ Web of Conferences, vol. 90, Article ID 01002, 6 pages, 2015.

[7] F.-H. Liu, Y.-Q. Gao, and B.-C. Li, "Comparing two-Boltzmann distribution and Tsallis statistics of particle transverse momentums in collisions at LHC energies," The European Physical Journal A, vol. 50, no. 8, article 123, 11 pages, 2014.

[8] M. D. Azmi and J. Cleymans, "Transverse momentum distributions at the LHC and Tsallis thermodynamics," Acta Physica Polonica B, Proceedings Supplement, vol. 7, no. 1, pp. 9-16, 2014.

[9] B. I. Abelev, J. Adams, M. M. Aggarwal et al., "Strange particle production in $p+p$ collisions at $\sqrt{s}=200 \mathrm{GeV}$," Physical Review $C$, vol. 75, no. 6, Article ID 064901, 21 pages, 2007.

[10] J. Adams, C. Adler, M. M. Aggarwal et al., "Identified hadron spectra at large transverse momentum in $\mathrm{p}+\mathrm{p}$ and $\mathrm{d}+\mathrm{Au}$ collisions at $\sqrt{s_{N N}}=200 \mathrm{GeV}$," Physics Letters B, vol. 637, pp. 161-169, 2006.

[11] A. Adare, S. Afanasiev, C. Aidala et al., "Nuclear modification factors of $\phi$ mesons in $d+\mathrm{Au}, \mathrm{Cu}+\mathrm{Cu}$, and $\mathrm{Au}+\mathrm{Au}$ collisions at $\sqrt{s_{N N}}=200 \mathrm{GeV}$," Physical Review C, vol. 83, Article ID 024909, 10 pages, 2011.

[12] A. Adare, S. Afanasiev, C. Aidala et al., "Identified charged hadron production in $p+p$ collisions at $\sqrt{s}=200 \mathrm{GeV}$ and 62.4 GeV," Physical Review C, vol. 83, Article ID 064903, 29 pages, 2011.

[13] B. Abelev, A. A. Quintana, D. Adamová et al., "Neutral pion and $\eta$ meson production in proton-proton collisions at $\sqrt{s}=0.9 \mathrm{TeV}$ and $\sqrt{s}=7$ TeV," Physics Letters B, vol. 717, no. 1-3, pp. 162-172, 2012.

[14] B. Abelev, J. Adam, D. Adamova et al., "Multi-strange baryon production in pp collisions at $\sqrt{s}=7 \mathrm{TeV}$ with ALICE," Physics Letters B, vol. 712, pp. 309-318, 2012.

[15] S. Chatrchyan, V. Khachatryan, A. M. Sirunyan et al., "Study of the inclusive production of charged pions, kaons, and protons in pp collisions at $\sqrt{\bar{s}}=0.9,2.76$, and7 TeV," The European Physical Journal C, vol. 72, article 2164, 37 pages, 2012.

[16] V. Khachatryan, A. M. Sirunyan, A. Tumasyan et al., "Transverse-momentum and pseudorapidity distributions of charged hadrons in $p p$ collisions at $\sqrt{s}=7 \mathrm{TeV}$," Physical Review Letters, vol. 105, no. 2, Article ID 022002, 14 pages, 2010.

[17] I. Sena and A. Deppman, "Systematic analysis of $p_{T}$-distributions in $\mathrm{p}+\mathrm{p}$ collisions," The European Physical Journal A, vol. 49, no. 2, article 17, 2013.

[18] S. Chatrchyan, V. Khachatryan, A. M. Sirunyan et al., "Study of the production of charged pions, kaons, and protons in $\mathrm{pPb}$ collisions at $\sqrt{s_{N N}}=5.02 \mathrm{TeV}$,' The European Physical Journal C, vol. 74, Article ID 2847, 27 pages, 2014.

[19] J. Cleymans and D. Worku, "The Tsallis distribution in protonproton collisions at $\sqrt{s}=0.9 \mathrm{TeV}$ at the LHC," Journal of Physics G: Nuclear and Particle Physics, vol. 39, no. 2, Article ID 025006, 12 pages, 2012. 
[20] M. D. Azmi and J. Cleymans, "Transverse momentum distributions in proton-proton collisions at LHC energies and Tsallis thermodynamics," Journal of Physics G: Nuclear and Particle Physics, vol. 41, no. 6, Article ID 065001, 10 pages, 2014.

[21] B.-C. Li, Y.-Z. Wang, and F.-H. Liu, "Formulation of transverse mass distributions in $\mathrm{Au}-\mathrm{Au}$ collisions at $\sqrt{S_{N N}}=$ $200 \mathrm{GeV} /$ nucleon," Physics Letters, Section B: Nuclear, Elementary Particle and High-Energy Physics, vol. 725, no. 4-5, pp. 352356, 2013.

[22] M. Rybczyński and Z. Włodarczyk, "Tsallis statistics approach to the transverse momentum distributions in $\mathrm{p}-\mathrm{p}$ collisions," The European Physical Journal C, vol. 74, article 2785, 5 pages, 2014.

[23] C.-Y. Wong and G. Wilk, "Tsallis fits to $p T$ spectra and multiple hard scattering in $p p$ collisions at the LHC," Physical Review D, vol. 87, no. 11, Article ID 114007, 19 pages, 2013.

[24] C.-Y. Wong and G. Wilk, "Tsallis fits to $p_{T}$ spectra for pp collisions at the LHC," Acta Physica Polonica B, vol. 43, no. 11, pp. 2047-2054, 2012.

[25] C.-Y. Wong, G. Wilk, L. J. L. Cirto, and C. Tsallis, "From QCDbased hard-scattering to nonextensive statistical mechanical descriptions of transverse momentum spectra in high-energy $p p$ and $p \bar{p}$ collisions," Physical Review D, vol. 91, Article ID 114027, 2015.

[26] H. Zhao and F.-H. Liu, "Chemical potentials of quarks extracted from particle transverse momentum distributions in heavy ion collisions at RHIC energies," Advances in High Energy Physics, vol. 2014, Article ID 742193, 14 pages, 2014.

[27] A. Adare, S. Afanasiev, C. Aidala et al., "Spectra and ratios of identified particles in $\mathrm{Au}+\mathrm{Au}$ and $d+\mathrm{Au}$ collisions at $\sqrt{s_{N N}}=$ 200 GeV,' Physical Review C, vol. 88, Article ID 024906, 16 pages, 2013.

[28] The CMS Collaboration, "Study of high- $p_{T}$ charged particle suppression in $\mathrm{PbPb}$ compared to $\mathrm{pp}$ collisions at $\sqrt{s_{N N}}=$ 2.76 TeV," The European Physical Journal C, vol. 72, Article ID 1945, 22 pages, 2012.

[29] B. I. Abelev, M. M. Aggarwal, Z. Ahammed et al., "Inclusive $\pi^{0}$, $\eta$, and direct photon production at high transverse momentum in $p+p$ and $d+$ Au collisions at $\sqrt{s_{N N}}=200 \mathrm{GeV}$," Physical Review C, vol. 81, no. 6, Article ID 064904, 26 pages, 2010.

[30] B. I. Abelev, M. M. Aggarwal, Z. Ahammed et al., "Energy dependence of $\pi^{ \pm}, p$ and $\bar{p}$ transverse momentum spectra for $\mathrm{Au}+\mathrm{Au}$ collisions at $\sqrt{s_{N N}}=62.4$ and $200 \mathrm{GeV}$," Physics Letters $B$, vol. 655, no. 3-4, pp. 104-113, 2007.

[31] B. I. Abelev, M. M. Aggarwal, Z. Ahammed et al., "Spectra of identified high- $p_{T} \pi^{ \pm}$and $p\left(\mathrm{p}^{-}\right)$in $\mathrm{Cu}+\mathrm{Cu}$ collisions at $\sqrt{{s_{N N}}}=$ 200 GeV,' Physical Review C, vol. 81, Article ID 054907, 8 pages, 2010.

[32] A. Adare, S. Afanasiev, C. Aidala et al., "Neutral pion production with respect to centrality and reaction plane in $\mathrm{Au}+\mathrm{Au}$ collisions at $\sqrt{s_{N N}}=200 \mathrm{GeV}$," Physical Review C, vol. 87, Article ID 034911, 19 pages, 2013.

[33] The ALICE Collaboration, "Production of charged pions, kaons and protons at large transverse momenta in $\mathrm{pp}$ and $\mathrm{Pb}-\mathrm{Pb}$ collisions at $\sqrt{s_{\mathrm{NN}}}=2.76 \mathrm{TeV}$," Physics Letters B, vol. 736, pp. 196-207, 2014.

[34] B. Abelev, J. Adam, D. Adamová et al., "Transverse momentum dependence of inclusive primary charged-particle production in $\mathrm{p}-\mathrm{Pb}$ collisions at $\sqrt{\mathrm{s}_{\mathrm{NN}}}=5.02 \mathrm{TeV}$," The European Physical Journal C, vol. 74, article 3054, 10 pages, 2014.

[35] B. I. Abelev, M. M. Aggarwal, Z. Ahammed et al., "Systematic measurements of identified particle spectra in $p p, d+\mathrm{Au}$, and
$\mathrm{Au}+\mathrm{Au}$ collisions at the STAR detector," Physical Review C, vol. 79, no. 3, Article ID 034909, 58 pages, 2009.

[36] F.-H. Liu, Y.-Q. Gao, T. Tian, and B.-C. Li, “Transverse momentum and pseudorapidity distributions of charged particles and spatial shapes of interacting events in $\mathrm{Pb}-\mathrm{Pb}$ collisions at 2.76 TeV," Advances in High Energy Physics, vol. 2014, Article ID 725739, 14 pages, 2014.

[37] C. Beck, "Non-extensive statistical mechanics and particle spectra in elementary interactions," Physica A: Statistical Mechanics and its Applications, vol. 286, no. 1, pp. 164-180, 2000.

[38] S. Chatrchyan, V. Khachatryan, A. M. Sirunyan et al., "Measurement of the $\Lambda_{b}$ cross section and the $\bar{\Lambda}_{b}$ to $\Lambda_{b}$ ratio with $J / \psi \Lambda$ decays in pp collisions at $\sqrt{s}=7 \mathrm{TeV}$," Physics Letters $B$, vol. 714, no. 2-5, pp. 136-157, 2012.

[39] S. S. Adler, S. Afanasiev, C. Aidala et al., "Centrality dependence of $\pi^{0}$ and $\eta$ production at large transverse momentum in $\sqrt{s_{N N}}=200 \mathrm{GeV} d+$ Au collisions," Physical Review Letters, vol. 98, no. 17, Article ID 172302, 7 pages, 2007.

[40] A. Adare, S. Afanasiev, C. Aidala et al., "Production of $\omega$ mesons in $p+p, d+\mathrm{Au}, \mathrm{Cu}+\mathrm{Cu}$, and $\mathrm{Au}+\mathrm{Au}$ collisions at $\sqrt{s_{N N}}=200$ GeV,' Physical Review C, vol. 84, Article ID 044902, 11 pages, 2011.

[41] A. Adare, S. Afanasiev, C. Aidala et al., "Transverse-momentum dependence of the $J / \psi$ nuclear modification in $d+$ Au collisions at $\sqrt{s_{N N}}=200 \mathrm{GeV}$,' Physical Review C, vol. 87, Article ID 034904, 11 pages, 2013.

[42] A. Adare, S. S. Adler, S. Afanasiev et al., "Direct photon production in $d+$ Au collisions at $\sqrt{s_{N N}}=200 \mathrm{GeV}$," Physical Review $C$, vol. 87, Article ID 054907, 8 pages, 2013.

[43] The ALICE Collaboration, "Multiplicity dependence of pion, kaon, proton and lambda production in $\mathrm{p}-\mathrm{Pb}$ collisions at $\sqrt{\mathrm{s}_{\mathrm{NN}}}=5.2$," Physics Letters B, vol. 728, pp. 25-38, 2014.

[44] M. M. Aggarwal, Z. Ahammed, A. V. Alakhverdyants et al., "Strange and multistrange particle production in $\mathrm{Au}+\mathrm{Au}$ collisions at $\sqrt{s_{N N}}=62.4 \mathrm{GeV}$," Physical Review C, vol. 83, no. 2, Article ID 024901, 16 pages, 2011.

[45] A. Adare, S. Afanasiev, C. Aidala et al., "Onset of $\pi^{0}$ suppression studied in $\mathrm{Cu}+\mathrm{Cu}$ collisions at $\sqrt{s_{N N}}=22.4,62.4$, and $200 \mathrm{GeV}$," Physical Review Letters, vol. 101, Article ID 162301, 6 pages, 2008.

[46] G. Agakishiev, M. M. Aggarwal, Z. Ahammed et al., "Strangeness enhancement in $\mathrm{Cu}-\mathrm{Cu}$ and $\mathrm{Au}-\mathrm{Au}$ collisions at $\sqrt{s_{N N}}=$ 200 GeV," Physical Review Letters, vol. 108, no. 7, Article ID 072301, 6 pages, 2012.

[47] A. Adare, S. Afanasiev, C. Aidala et al., " $J / \psi$ production in $\sqrt{s_{N N}}=200 \mathrm{GeV} \mathrm{Cu}+\mathrm{Cu}$ collisions," Physical Review Letters, vol. 101, Article ID 122301, 6 pages, 2008.

[48] B. I. Abelev, M. M. Aggarwal, Z. Ahammed et al., "Identified baryon and meson distributions at large transverse momenta from $\mathrm{Au}+\mathrm{Au}$ Collisions at $\sqrt{s_{N N}}=200 \mathrm{GeV}$,' Physical Review Letters, vol. 97, no. 15, Article ID 152301, 6 pages, 2006.

[49] S. S. Adler, S. Afanasiev, C. Aidala et al., "Common suppression pattern of $\eta$ and $\pi^{0}$ mesons at high transverse momentum in $\mathrm{Au}+\mathrm{Au}$ collisions at $\sqrt{\mathrm{s}_{\mathrm{NN}}}=200 \mathrm{GeV}$," Physical Review Letters, vol. 96, Article ID 202301, 2006.

[50] L. Adamczyk, G. Agakishiev, M. M. Aggarwal et al., " $J / \Psi$ production at high transverse momenta in $\mathrm{p}+\mathrm{p}$ and $\mathrm{Au}+\mathrm{Au}$ collisions at $\sqrt{s_{N N}}=200 \mathrm{GeV}$,' Physics Letters B, vol. 722, no. $1-3$, pp. 55-62, 2013.

[51] S. Afanasiev, C. Aidala, N. N. Ajitanand et al., "Measurement of direct photons in $\mathrm{Au}+\mathrm{Au}$ collisions at $\sqrt{s_{N N}}=200 \mathrm{GeV}$," Physical Review Letters, vol. 109, Article ID 152302, 7 pages, 2012. 
[52] PHENIX Collaboration, "Centrality dependence of lowmomentum direct-photon production in $\mathrm{Au}+\mathrm{Au}$ collisions at $\sqrt{{s_{N N}}}=200 \mathrm{GeV}$," Physical Review C, vol. 91, Article ID 064904, 11 pages, 2015.

[53] B. Abelev, J. Adam, D. Adamová et al., " $K^{*}(892)^{0}$ and $\phi(1020)$ production in $\mathrm{Pb}-\mathrm{Pb}$ collisions at $\sqrt{s_{N N}}=2.76 \mathrm{TeV}$," Physical Review C, vol. 91, Article ID 024609, 26 pages, 2015.

[54] B. Abelev, J. Adam, D. Adamová et al., " $K_{S}^{0}$ and $\Lambda$ production in $\mathrm{Pb}-\mathrm{Pb}$ collisions at $\sqrt{s_{N N}}=2.76 \mathrm{TeV}$," Physical Review Letters, vol. 111, Article ID 222301, 10 pages, 2013.

[55] The ALICE Collaboration, "Multi-strange baryon production at mid-rapidity in $\mathrm{Pb}-\mathrm{Pb}$ collisions at $\sqrt{s_{N N}}=2.76 \mathrm{TeV}$," Physics Letters B, vol. 728, pp. 216-227, 2014.

[56] A. Banerjee and V. M. Yakovenko, "Universal patterns of inequality," New Journal of Physics, vol. 12, Article ID 075032, 25 pages, 2010. 

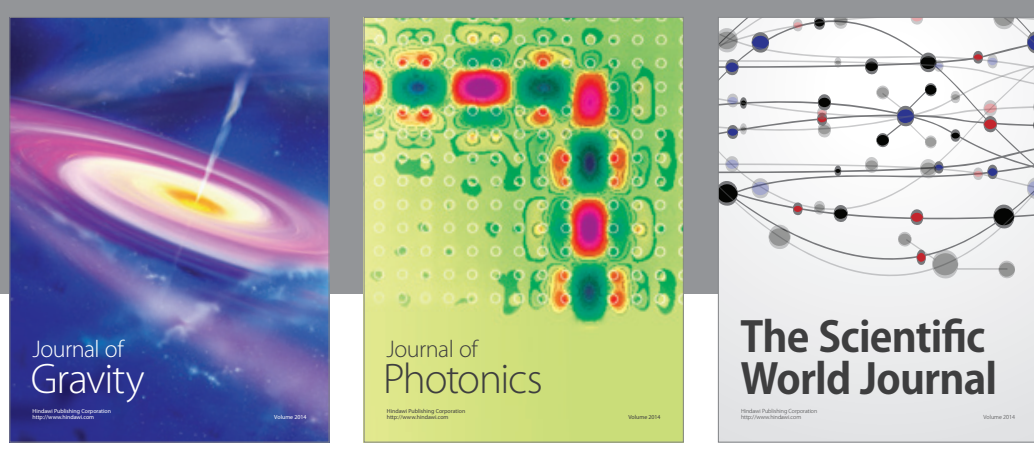

The Scientific World Journal
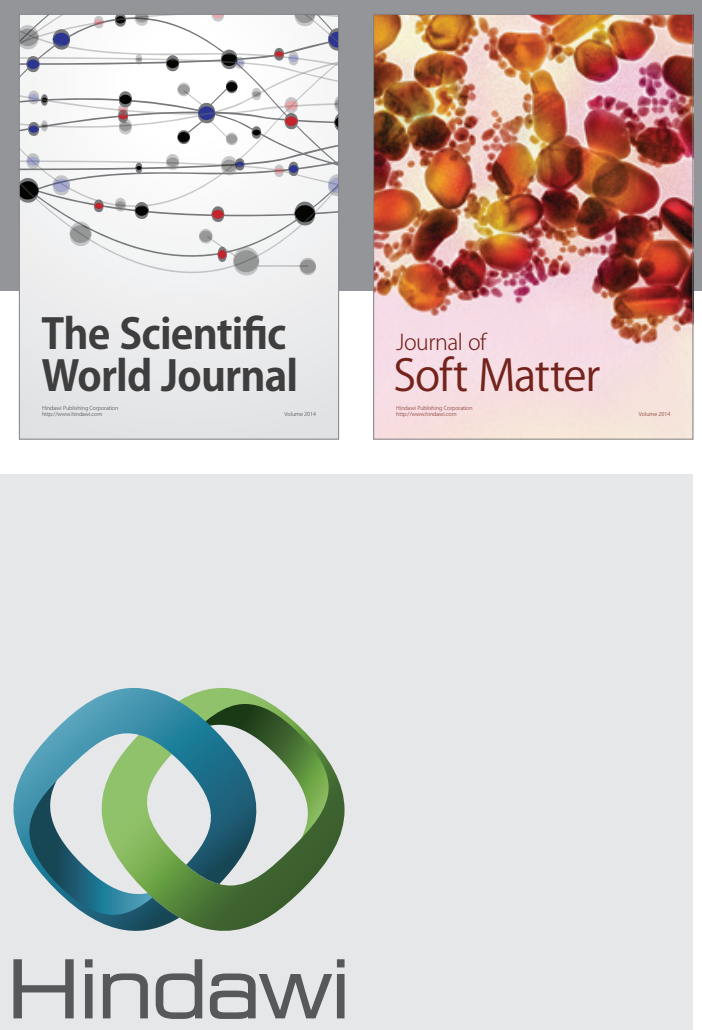

Submit your manuscripts at

http://www.hindawi.com

nternational Journal of

Statistical Mechanics
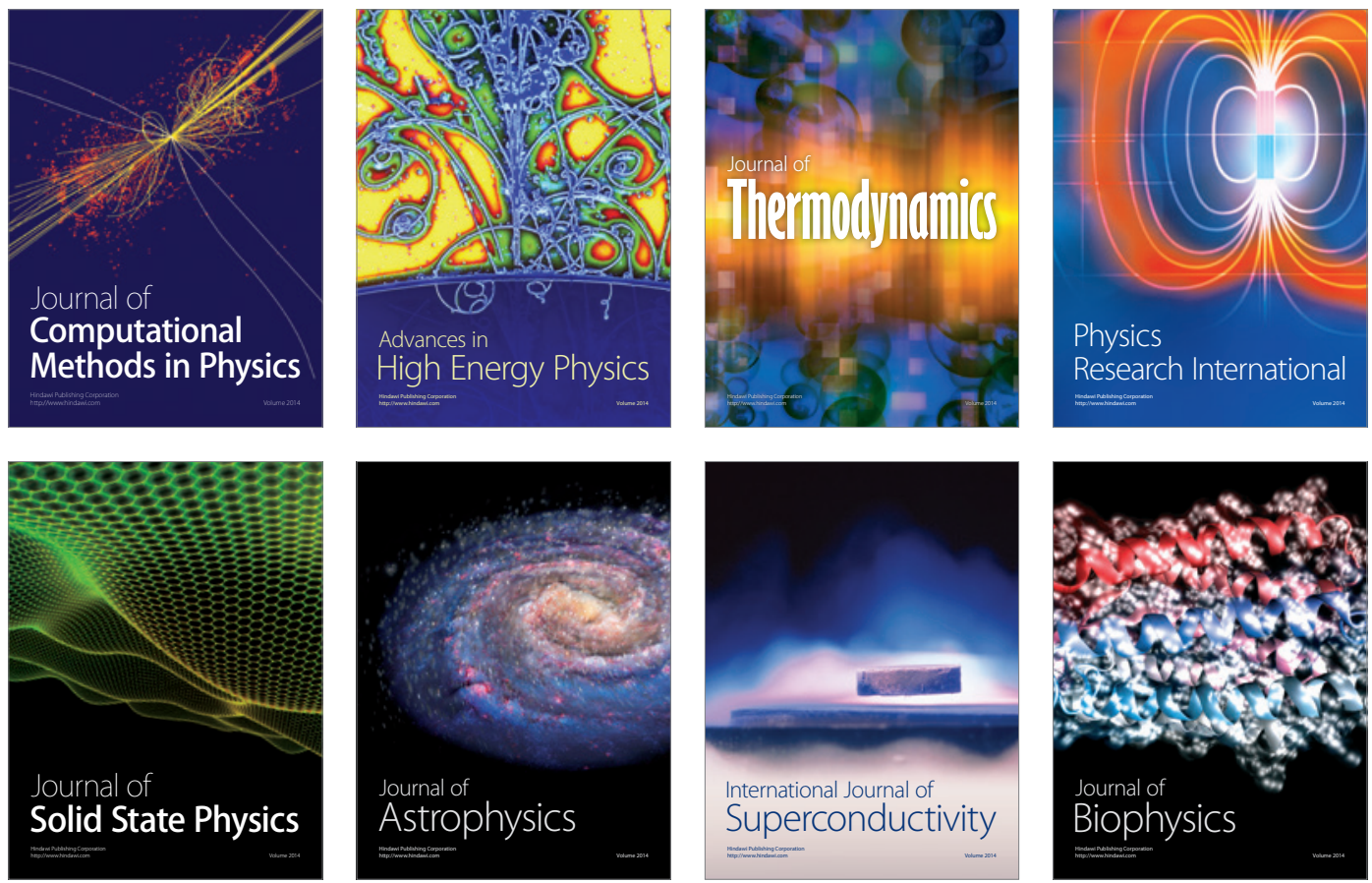
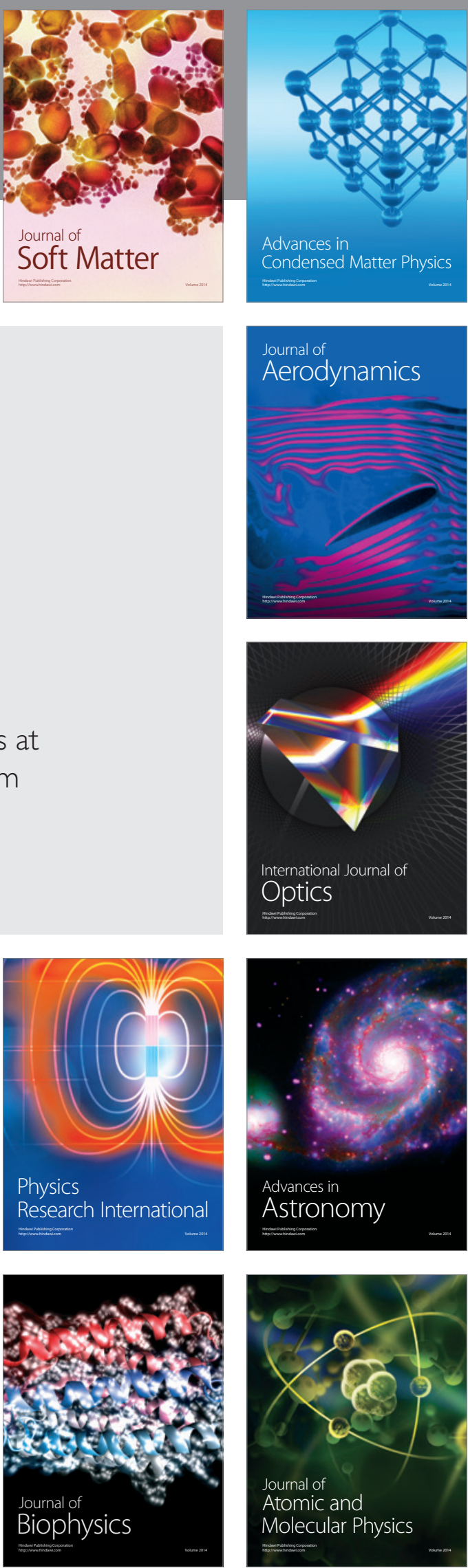